\title{
Departamento de Neurologia, Psiquiatria e Psicologia Médica
}

\author{
Cristina M. Del Bem; Michel P. Lison \& Edna M. Marturano \\ Docentes. Departamento de Neurologia, Psiquiatria e Psicologia Médica. Faculdade de Medicina de Ribeirão Preto - USP
}

$\mathrm{O}$ Departamento de Neurologia, Psiquiatria e Psicologia Médica é o resultado da fusão de 2 departamentos, o de Neurologia e o de Psicologia Médica e Psiquiatria. Historicamente, é possível distinguir no departamento três setores amplos, que se desenvolveram com projetos próprios ao longo do tempo. Esses setores correspondem às disciplinas de Neurologia, Psiquiatria e Psicologia. $\mathrm{O}$ relato que segue obedece a essa imposição histórica.

\section{AS PRIMEIRAS DÉCADAS}

\subsection{Neurologia}

A história da Neurologia na FMRP já integra os anais da Neurologia no Brasil. A maior parte das informações relatadas nos próximos parágrafos foi extraída do capítulo escrito por Lison ${ }^{(1)}$ para a História da Neurologia no Estado de São Paulo.

As atividades do Departamento de Neurologia, antes da fusão com o de Psiquiatria e Psicologia Médica, foram dirigidas, desde 1956, por Paulo Pinto Pupo, Professor Livre-Docente da Faculdade de Medicina da USP e Chefe da Clínica Neurológica da Escola Paulista de Medicina, convidado, naquele ano, por Zeferino Vaz para criar e dirigir o Departamento de Clínica Neurológica. No mesmo ano de 1956, Paulo Pupo ministrou a disciplina de Neurologia, tendo como assistente Mário Martinez e organizou o departamento. Para o laboratório de líquor, Pupo convidou Isnard dos Reis Filho, que conhecia da Escola Paulista de Medicina. Já em 1956, o novo docente começou a realizar exames de líquor no Laboratório Central do Hospital das Clínicas, enquanto Mário Martinez iniciou atividades de encefalografia junto ao Departamento de Fisiologia, onde havia equipamento para isso. Só em 1960, com aparelhagem emprestada pela Escola Paulista de Medicina, foi possível a Mário Martinez instalar um serviço de EEG, próprio do Departamento de Neurologia, no antigo HC. As atividades de Neurorradiografia estavam a cargo de Sylvio de Vergueiro Forjaz, que as desempenhava junto ao Setor de Neurocirurgia ${ }^{(2)}$.

Com a saída de Paulo Pinto Pupo, a chefia do Departamento de Neurologia foi confiada, em fins de 1958, a Jorge Armbrust de Lima Figueiredo, então docente da Escola Paulista de Medicina e convidado por Zeferino Vaz como catedrático contratado. No início de 1959, o departamento convidou Edymar Jardim, formado pela Escola Paulista de Medicina em 1958, para auxiliar de ensino e incumbido de reinstalar o laboratório de líquor, que se desativara com a saída de Isnard dos Reis Filho.

Em 1960, incorporou-se ao corpo docente, como instrutor, Rubens de Moura Ribeiro, proveniente da E.P.M. No ano seguinte, Michel Pierre Lison foi contratado para exercer atividades em Neurologia Experimental.

Seguiu-se um período de implementação de serviços para fins assistenciais, pedagógicos e de pesquisa, apoiados, quando necessário, por outros departamentos. Destes destacam-se o de Patologia e o de Morfologia Humana, onde puderam se desenvolver pesquisas experimentais nos domínios da Doença de Chagas (Edymar Jardim) e das alterações dos neurô- 
nios após a secção do axônio, através de métodos citoquímicos quantitativos (Michel Pierre Lison), que resultaram na elaboração das duas primeiras teses de doutoramento da área, defendidas em dezembro de 1962.

A expansão dos serviços foi rápida: inauguração, em 1961, das enfermarias de Clínica Neurológica do Hospital das Clínicas da Faculdade de Medicina de Ribeirão Preto (22 leitos), assunção da responsabilidade por seis leitos para a Neuropediatria, inicialmente no Departamento de Pediatria, criação da Residência Médica em Neurologia, em 1962, aquisição e instalação do eletroencefalógrafo próprio, substituindo aparelho emprestado da Escola Paulista de Medicina, instalado em 1959. Iniciativas de docentes surgiram desde o início da década de 60, como as de Jardim, de engajar-se nas técnicas neurorradiológicas, prestando serviços assistenciais e de ensino aos primeiros médicos residentes, além de praticar exames eletromiográficos

Dos primeiros trabalhos publicados pelo departamento, no início da década de 1960, destacam-se três, de Forjáz e Martinez, em co-autoria, versando sobre formas obstrutivas de neurocisticercose. A elevada incidência da parasitose na região levaria a uma seqüência de trabalhos clínicos que se estenderia até os dias presentes. A preocupação do serviço com esse grave problema de saúde pública da região foi fator determinante da publicação do Decreto Municipal $n^{\circ}$ 397, de 20.10.92, considerando a neurocisticercose doença de notificação compulsória.

Durante 1962, foram publicados 12 trabalhos em revistas nacionais e estrangeiras. Entretanto, em 1963, não foi possível manter a mesma produção, em conseqüência do crescimento do departamento e do aumento das atividades assistenciais de rotina. Pesquisas já iniciadas foram interrompidas. A exoneração, de Mário L. Martinez, no início de 1963, agravou o problema. O reflexo dessa situação foi o remanejamento interno da disciplina, voltado mais para estruturação de serviços clínicos diversificados do que para o aprimoramento científico do corpo docente e cujos efeitos perduraram até o final da década de 80. Assim, Lison abandonou seus projetos em Neurologia Básica, incluindo viagem ao Exterior, passando a atuar no Serviço de Eletroencefalografia e iniciando trabalhos em eletroencefalografia clínica e epilepsia. Estruturou-se o Serviço de Neurologia Infantil, sob a responsabilidade de Maria Valeriana Moura Ribeiro, contratada pelo departamento em 1963 e, a partir de
1990, de Carolina Araújo Rodrigues Funayama. Desenvolveu-se projeto de correlação clínico-patológica em afecções do sistema nervoso para fins científicos e reuniões clínico-patológicas regulares a partir de 1960, ao encargo de Richardo Antonio Gallina, transferido para o Departamento em 1967.

A projeção de departamento, na época, justificou a realização, em Ribeirão Preto, do I Congresso Brasileiro de Neurologia, em julho de 1964, sob a presidência de Adherbal Tolosa, cabendo a organização ao Departamento de Neurologia.

As atividades de pesquisa em Neurologia foram desenvolvidas com base em recursos locais. Nas duas primeiras décadas, não houve possibilidade de estágios pós-doutorais em centros de renome internacional. A preocupação dominante era a de estruturar o serviço. O departamento, mesmo com essa falha, ganhou renome nos meios científicos nacionais, particularmente nas áreas de Neurologia Tropical, Neurologia Infantil, Eletroencefalografia e Epilepsias.

Em 1962, foi instalada a Residência em Neurologia, cujo primeiro preceptor foi Lison e, posteriormente, as Residências em Neurofisiologia Clínica e Neurologia Infantil. Tais atividades possibilitaram o exercício da especialidade pela maioria dos profissionais que atuam em Ribeirão Preto e região assim como a obtenção de título de especialista em Neurologia e/ou Eletroencefalografia por significativo número de antigos residentes.

Em junho de 1971, foi autorizado Curso de PósGraduação em Neurologia, em nível de Mestrado e Doutorado, o primeiro do país na especialidade. Lison, por delegação da chefia do departamento, elaborou o projeto e foi responsável por sua coordenação até 1980. O curso foi credenciado pelo Conselho Federal de Educação em setembro de 1975.

Em novembro de 1980, realizou-se, em Ribeirão Preto, o VII Congresso Brasileiro de Eletroencefalografia e Neurofisiologia Clínica, sob a Presidência de Lison. Tratava-se de experiência ousada por ser o primeiro congresso realizado independentemente do da Academia Brasileira de Neurologia e o primeiro a ser realizado numa cidade do interior, pouco mais de um mês após o IX Congresso Brasileiro de Neurologia, em Curitiba. O bom nível de organização, o rendimento científico e o comparecimento de cerca de 300 participantes, asseguraram o sucesso do evento, do qual dependia a preservação da autonomia da Sociedade Brasileira de Eletroencefalografia e Neurofisiologia Clínica. 
No início da década de 80 o setor de Neurologia encontrava-se limitado em sua capacidade técnica e operacional. O ambiente ainda era estimulante, mas ameaçado por um processo de acomodação e de distanciamento dos progressos nos diversos domínios, agravado pelo isolamento internacional. Nessa conjuntura, foi de fundamental importância a concessão de afastamento a Amilton Antunes Barreira para estagiar no Serviço do Prof. Lapresle em Paris, entre 1980 e 1982. Na França, Barreira adquiriu conhecimentos avançados na área das neuropatias periféricas e da tomografia computadorizada graças aos contatos mantidos com Gérard Said e Odile Missir e, posteriormente, com especialistas norte-americanos e ingleses. No seu retorno, Barreira assumiu o gerenciamento do Setor de Tomografia Computadorizada, da Unidade de Emergência e a direção do Laboratório de Neurologia Aplicada e Experimental.

Regina Maria França Fernandes, docente contratada após estágio nos Estados Unidos, implantou estudos poligráficos e eletroencefalográficos no período neonatal.

Américo Ceiki Sakamoto obteve afastamento para ir ao exterior pelo maior tempo até então concedido pela FMRP-USP: 4 anos (1988-1990) para estagiar junto à Seção de Epilepsia e Neurofisiologia Clínica da Cleveland Clinic Foundation e em 1990-1992 na VBA Bethec, Bielefeld, Alemanha. Em seu retorno, organizou o CIREP-Centro de Cirurgia de Epilepsias - cujas atividades iniciaram-se em 1994. Os objetivos que norteiam o funcionamento do CIREP são o oferecimento de um atendimento integral com equipe multiprofissional e interdisciplinar aos portadores de epilepsia e aos seus familiares, visando ao alívio das crises, à reabilitação e à maior reintegração social, com redução do estigma associado à epilepsia, proporcionando uma melhor qualidade de vida.

O preparo profissional e acadêmico dos docentes tem sido de fundamental importância para a continuidade e crescimento da contribuição do departamento à Neurologia Brasileira. Mais recentemente, as contratações de Wilson Marques Júnior e João Pereira Leite, ambos com pós-doutorado no Exterior, contribuíram, também, para reafirmar a posição do grupo na investigação científica de importantes temas de fronteira na Neurologia.

\subsection{Psiquiatria}

Em sua origem, o setor constituía o Departamento de Psicologia Médica, depois denominado Departamento de Psiquiatria e Psicologia Médica.
Os fundamentos iniciais do Departamento de Psicologia Médica foram delineados pelo psiquiatra e psicanalista chileno, Sérgio Rodriguez Gonzáles, que assumiu o departamento em 1956, a convite do então diretor e fundador da Faculdade de Medicina de Ribeirão Preto, professor Zeferino Vaz.

No ano seguinte, o professor Sérgio Gonzáles foi substituído na chefia do departamento, pelo seu conterrâneo e também psicanalista Prof. Dr. Hernan Ramon Davanzo Corte, que permaneceu no Brasil até o ano de 1965.

No início de suas atividades no departamento, o professor Davanzo contava apenas com uma assistente, a psicóloga Yone Neves Graca. Progressivamente, novos profissionais, como os doutores David Azoubel Neto, Lenise Lisboa Azoubel, Caiuby M de Azevedo Trench, Conceição Ribeiro da Costa, Clotilde Rossetti Ferrreira e Wilma Ribeiro, foram sendo incorporados ao departamento. Alguns egressos das primeiras turmas da Faculdade de Medicina também foram absorvidos pelo departamento, como os doutores Hona Thaim Mantovani, Flávio D’Andrea e Rachel Vilela Fávero.

A gestão do professor Davanzo se caracterizou pela adoção de uma linha teórica de orientação psicanalítica, pela ênfase em trabalho multidisciplinar, com uma equipe composta por psiquiatras, psicólogos, terapeutas ocupacionais, assistentes sociais e enfermeiros psiquiátricos e pela implantação de programas terapêuticos voltados para o atendimento extra-hospitalar e para a reabilitação social.

Já no ano de 1959, o Prof. Davanzo coordenava um grupo de atividades terapêuticas, denominado Clube da Alvorada, que se caracterizava pela realização de atividades recreativas e sociais com finalidades terapêuticas. Esse clube foi precursor do programa de internação parcial, cujo projeto de implantação inicial fracassou em 1961 devido à falta de espaço físico e de pessoal. O serviço foi instalado definitivamente no ano de 1965, o que foi possível porque, entre outras coisas, a Faculdade de Medicina adquiriu um prédio situado à avenida Nove de Julho, onde funcionava o Hospital Psiquiátrico Emboaba. Em 1967, as atividades do Hospital Dia foram interrompidas, permanecendo apenas o atendimento em terapia ocupacional. Em 1974, o Hospital Dia foi reativado e mantém atendimento até os dias de hoje.

No final do ano de 1961, foram instalados 4 leitos psiquiátricos no antigo Hospital das Clínicas (atual Unidade de Emergência), o que poderia ser caracterizado como o embrião de uma das primeiras enferma- 
rias psiquiátricas em hospital geral, no Brasil. Isso certamente significava um grande avanço para a época, porque, mesmo nos dias de hoje, sabe-se que a grande maioria dos leitos psiquiátricos ainda está em hospitais psiquiátricos e não em hospitais gerais. Infelizmente, tais leitos foram desativados 1 ano e 8 meses após sua inauguração.

Também durante o citado período, foram criados serviços ambulatoriais. O Serviço de Psiquiatria Infantil iniciou suas atividades no início da década de 1960 e, até hoje, desenvolve atendimento ludoterápico, medicamentoso e de orientação familiar, em um modelo multidisciplinar. Em 1962, iniciaram-se as atividades do ambulatório de psicoterapia de orientação psicanalítica, que até hoje funciona no prédio da Nove de Julho.

Com a saída do Prof. Davanzo, o Departamento de Psicologia Médica e Psiquiatria foi chefiado interinamente por docentes dos departamentos de Clínica Médica e Medicina Social, até que, em 01/04/69, houve a fusão com o Departamento de Neurologia ${ }^{(2)}$, constituindo-se o Departamento de Neuropsiquiatria e Psicologia Médica, posteriormente rebatizado com sua denominação atual.

No início da década de 70 o professor Luiz da Rocha Cerqueira foi convidado para chefiar o setor de Psiquiatria. O Prof. Cerqueira havia atuado, há pouco, como Coordenador de Saúde Mental do governo do Estado de São Paulo, e era defensor da necessidade de implantação de mudanças na assistência psiquiátrica, calcada, basicamente no asilamento dos portadores de transtorno mental em grandes manicômios psiquiátricos. Foi um período que se caracterizou pela instalação de serviços e políticas baseadas em um modelo de Psiquiatria Comunitária. O Prof. Cerqueira pôde contar com a colaboração ativa do então recémcontratado, professor José Onildo Betioli Contel.

No ano de 1972, foi estabelecido um convênio entre a Faculdade de Medicina de Ribeirão Preto e a Secretaria Estadual de Saúde para a implantação de um Programa de Saúde Mental na região, a ser executado pela disciplina de Psiquiatria. Através desse convênio, a disciplina de Psiquiatria pôde ampliar suas atividades, através da contratação de vários profissionais, o que permitiu a criação de serviço ambulatorial, vinculado ao Hospital Psiquiátrico de Ribeirão Preto (atual Hospital Santa Tereza), além do acompanhamento de pacientes internados na instituição.

Muitos dos profissionais contratados nessa época, como os doutores Sonia Regina Loureiro, José
Hércules Golfeto, Maria Auxiliadora Campos, Cláudio Roberto Carvalho Rodrigues, Gerson Antonio Vansan, tornaram-se, posteriormente, docentes da disciplina.

O referido programa tinha uma duração prevista de 5 anos. Embora o convênio com a universidade tenha terminado, os serviços instituídos permaneceram, sendo que o ambulatório instalado na época, no hospital psiquiátrico, foi o embrião do Ambulatório Regional de Saúde Mental, atualmente gerenciado pela Prefeitura Municipal de Ribeirão Preto.

Outro exemplo é o Serviço de Avaliação Psicodiagnóstica, que embora tenha tido suas atividades suspensas em dezembro de 1977, com o fim do convênio, foi reativado em 1981, com a contratação de uma psicóloga, Sonia Regina Loureiro, para compor o quadro de docentes da disciplina de Psiquiatria.

Durante esse período, a disciplina ampliou também suas atividades no próprio Hospital das Clínicas, com o início, em 1975, das atividades do serviço de emergências psiquiátricas. Atualmente, esse é o único serviço de emergências psiquiátricas da região, sendo a referência para o atendimento de 25 municípios com uma população estimada em 1.200.000 habitantes.

Em 1978, foi inaugurado o novo prédio do Hospital das Clínicas no Campus de Ribeirão Preto, sendo parte do terceiro andar destinada à Psiquiatria. Naquele ano foi inaugurada a Enfermaria de Psiquiatria, que funciona até os dias de hoje com 16 leitos. Recentemente, foram ativados mais 8 leitos no andar, destinados a internações breves.

O Serviço de Interconsultoria Psiquiátrica se consolidou e passou a se desenvolver de maneira sistematizada, a partir da mudança para o novo prédio e, desde então, vem oferecendo atendimento a pacientes internados nas enfermarias de outras especialidades.

Também nessa época, os serviços ambulatoriais se expandiram com a instalação do Ambulatório de Clínica Psiquiátrica, que até os dias de hoje, funciona em conjunto com os demais ambulatórios da instituição, oferecendo atendimento psicoterápico e farmacológico em diferentes programas.

Tal diversidade de serviços permitiu que fosse oferecido, para alunos de graduação e de especialização, um treinamento calcado em modelos avançados de assistência psiquiátrica, baseado no atendimento multidisciplinar. Esse treinamento inclui médicos, psicólogos, assistentes sociais e terapeutas ocupacionais. A Residência Médica em Psiquiatria foi instituída em 1963, contando com apenas uma vaga. Com o passar 
dos anos, o número de vagas foi aumentando progressivamente, até chegar a 10 vagas para o primeiro ano, em 1979, o que se mantém até os dias de hoje. Além do primeiro e segundo anos obrigatórios, ainda existem programas a serem desenvolvidos em um terceiro ano opcional.

Para profissionais não médicos, o treinamento é vinculado ao Programa de Aprimoramento Profissional na Área da Saúde, coordenado pela FUNDAP. A Divisão de Psiquiatria oferece cursos de aprimoramento em Psicologia/Saúde Mental, Terapia Ocupacional em Psiquiatria e Serviço Social em Psiquiatria.

Em síntese, a história da Psiquiatria, na FMRPUSP caracterizou-se pela ênfase na implantação de modelos de assistência psiquiátrica, com repercussões na formação de recursos humanos e na extensão de serviços à comunidade. Provavelmente, a organização de serviços assistenciais de qualidade tenha um impacto muito maior na Psiquiatria do que em qualquer outra especialidade médica. Tradicionalmente, $\mathrm{o}$ atendimento psiquiátrico se concentrava em grandes hospitais psiquiátricos, desvinculados da prática médica geral. Apesar de inúmeros esforços técnicos, é o modelo que ainda prevalece em muitas regiões do país. A disciplina de Psiquiatria da FMRP-USP assumiu um papel relevante na implantação de novas políticas de Saúde Mental na Região de Ribeirão Preto, contribuindo ativamente para a criação de uma rede de serviços de Saúde Mental, hierarquizada e descentralizada, que oferece atendimento tecnicamente eficiente.

\subsection{Psicologia}

\subsubsection{Primórdios}

A história da Psicologia, no departamento, começa com a Profa. Maria Clotilde Rossetti-Ferreira, que deu início a uma linha de pesquisa sobre desenvolvimento da criança pequena, tendo obtido da FAPESP um dos primeiros auxílios por ela concedidos, em meados da década de 60, para a montagem do laboratório de observação do comportamento, ainda em uso no prédio da Av. 9 de Julho. O grupo de pesquisa, então constituído pela Profa. Clotilde, desenvolveu, no departamento, diversos projetos, que resultaram em teses de doutorado defendidas no Instituto de Psicologia da USP.

As atividades de pesquisa, ensino e extensão teriam novo impulso com a curta porém profícua participação da Profa. Thereza Pontual de Lemos Mettel no quadro docente do departamento. A Profa. Mettel, que realizara seu treinamento clínico e de pesquisa em universidades norte-americanas, foi uma das primeiras psicólogas brasileiras a trabalhar com Psicologia Hospitalar. Iniciou as práticas de interconsultas e $\mathrm{o}$ atendimento ambulatorial de crianças, abrindo oportunidades de estágio para alunos de Medicina e Psicologia. A iniciação profissional que proporcionava aos estagiários, baseada em princípios de modificação do comportamento, tinha a marca do scientistpractitioner, aquele que avalia continuamente suas práticas através de técnicas objetivas. Entre os primeiros estagiários recebidos pela Profa. Mettel estavam os depois professores Antonio Waldo Zuardi, Edna Maria Marturano e Ricardo Gorayeb.

Contemporâneo das Profas. Clotilde e Mettel, no departamento, o Prof. João Cláudio Todorov, que anos depois se tornaria reitor da $\mathrm{UnB}$, trabalhava com pesquisa básica em análise experimental do comportamento, tendo colaborado na criação do Laboratório Integrado de Psicologia e Farmacologia, juntamente com o Prof. Frederico Guilherme Graeff, então docente da FMRP.

\subsubsection{Pesquisa e Pós-Graduação: O Intercâmbio com Outras Unidades}

Os três docentes citados acima deram início a uma produtiva participação em programas de pós-graduação de outras unidades, notadamente nos cursos de Mestrado e Doutorado em Psicologia Experimental do Instituto de Psicologia da USP. Esse intercâmbio foi mantido pelos professores Isaías Pessotti e Edna Maria Marturano até meados da década de 90, quando a criação de dois novos cursos da área no Campus de Ribeirão Preto demandou maior participação nos programas locais. Porém foi na UFSCar que a colaboração de docentes do departamento se fez mais presente. No final da década de 70, o Centro de Educação e Ciências Humanas daquela universidade criou o Curso de Pós-graduação em Educação Especial, de cuja organização o Prof. Pessotti participou ativamente. Todos os docentes da Psicologia da FMRP, inclusive o recém-doutorado Prof. Ricardo Gorayeb, colaboraram com esse programa no período de sua implantação, orientando dissertações e ministrando disciplinas.

Mais recentemente, com a criação do Curso de Pós-Graduação em Psicologia da FFCLRP, os docentes do departamento, vinculados à disciplina Psicologia foram convidados a integrar o quadro de orientadores daquele programa, estando nele credenciados os professores Edna Maria Marturano, Maria Beatriz Martins Linhares e Ricardo Gorayeb. 


\subsubsection{Extensão de Serviços à Comunidade}

Ao passo que, por mais de duas décadas, a pesquisa e a produção intelectual dos docentes da Psicologia estiveram associadas a programas de pós-graduação de outras instituições de ensino superior, no âmbito do departamento, sempre houve oportunidade e apoio para a extensão de serviços à comunidade, seja mediante a criação de unidades prestadoras de serviços ou a oferta de programas de formação de recursos humanos para atuação profissional na área da saúde. Essa tendência se consolidou a partir de 1974, quando o quadro de docentes se estabilizou.

Em 1975, foi organizado o Ambulatório de Psicologia Infantil e, no mesmo ano, por sugestão do chefe do departamento, Prof. Jorge Armbrust de Lima Figueiredo, os docentes elaboraram um projeto de Residência em Psicologia Clínica Infantil, o primeiro no país, nos moldes da Residência Médica, e redigiram seu regulamento. Embora com alteração do nome, o programa foi aprovado pelo Conselho Deliberativo do Hospital das Clínicas da FMRP e passou a funcionar a partir de 1976, subsidiado com bolsas e tendo como preceptores os professores Edna e Gorayeb. Esse programa foi a base para a regulamentação dos Programas de Aprimoramento, tanto no $\mathrm{HC}$ de Ribeirão Preto como em todo o Estado de São Paulo. Passou a servir de modelo para diversos hospitais, inclusive para a FUNDAP, que, em 1987, publica o Manual de Procedimentos do Programa de Aprimoramento na Área da Saúde, inspirado no Regulamento dos Cursos de Aprimoramento para Profissionais Não Médicos do HCFMRP.

Atualmente, a disciplina mantém a Residência em Psicologia Clínica e os Cursos de Aprimoramento em Psicologia do Desenvolvimento na Área da Saúde, Promoção de Saúde na Comunidade e Psicopedagogia. Esses programas foram viabilizados com a criação de serviços de Psicologia Ambulatorial e Hospitalar, coordenados por docentes, que servem como campo de treinamento dos aprimorandos, sob a supervisão direta de psicólogos assistentes, contratados pelo HC.

\subsubsection{A Sociedade Brasileira de Psicologia}

A história da Sociedade Brasileira de Psicologia foi construída com a colaboração efetiva de docentes vinculados ao departamento. Nos seus primórdios, ainda quando Sociedade de Psicologia de Ribeirão Preto, teve como presidentes os professores Isaías
Pessotti e Ricardo Gorayeb, este por diversas gestões, tendo sido um grande incentivador do reconhecimento da antiga SPRP como associação representativa da Psicologia, no Brasil. E foi sob a presidência do Prof. Gorayeb que, em 1991, a SPRP foi transformada na Sociedade Brasileira de Psicologia, tendo hoje sua representatividade reconhecida nacional e internacionalmente, como interlocutora de órgãos oficiais, como o CNPq, e de associações internacionais, como a SIP.

\section{O MOMENTO ATUAL: TENDÊNCIAS E PERSPECTIVAS}

$\mathrm{Na}$ atualidade, duas tendências dominantes podem ser identificadas nas atividades do Departamento de Neurologia, Psiquiatria e Psicologia Médica: a interdisciplinaridade e a ênfase na investigação científica. Ambas as tendências se concretizam através dos programas de pós-graduação oferecidos pelo departamento: o Programa de Pós-Graduação em Neurologia, iniciado em 1971, e o Programa de Pós-Graduação em Saúde Mental, iniciado em 1991.

\subsection{Pós-Graduação em Neurologia}

O programa de Pós-Graduação em Neurologia teve início em 15 de junho de 1971. Foi credenciado pelo Conselho Federal de Educação em 1975 e recredenciado no ano de 1985. Nos seus 30 anos de existência, titulou dezenas de doutores e mestres que hoje atuam principalmente em universidades públicas do país ${ }^{(1)}$.

O programa era, inicialmente, destinado a médicos neurologistas mas, a partir de 2001, passou a abranger duas áreas de concentração: 1) Neurologia, para médicos neurologistas (que tenham já, no mínimo, 2 anos de residência médica em Neurologia); 2) Neurociências, para médicos não especializados em Neurologia e profissionais não médicos de áreas biológicas, ou cujo interesse investigativo envolva as neurociências. Em ambas as áreas, ele é desenvolvido nos níveis de Mestrado e Doutorado. Este é reservado para alunos com Mestrado prévio ou com comprovada experiência em docência e pesquisa científica. Os programas de Mestrado e Doutorado foram elaborados de tal forma que metade dos créditos sejam obtidos junto às disciplinas do programa. Todas as linhas de pesquisa têm, no mínimo, uma disciplina correspondente. Parte dos créditos pode ser obtida em disciplinas de outros programas, conforme o interesse 
do aluno. Para a execução dos projetos de pesquisa, feitos sob orientação de docentes ligados ao programa, existe disponibilidade de diferentes laboratórios, cujas atividades variam de análises neurofisiológicas à Biologia Molecular, biotério para trabalhos com animais de experimentação e de acesso a pacientes do Hospital das Clínicas (HCRP) da FMRP-USP.

Os estudos clínicos são feitos nos ambulatórios e enfermarias de Neurologia. A enfermaria tem leitos para pacientes eletivos, havendo leitos específicos para: Neurologia Geral, Neurologia Infantil, Doenças Neuromusculares, Doenças Vasculares do Sistema Nervoso, Epilepsia, Neurogenética. O CIREP - Centro de Cirurgia de Epilepsia, faz videomonitorizações contínuas de pacientes epilépticos para seleção de casos cirúrgicos. Na Unidade de Emergência do Hospital das Clínicas, há leitos para atendimento e investigação de urgências e emergências neurológicas.

O programa mantém relacionamento formal e informal com outras clínicas e laboratórios do Hospital das Clínicas de Ribeirão Preto e do Campus e de outros centros acadêmicos do país e do exterior, nos Estados Unidos da América do Norte e na Europa. Tal relacionamento é particularmente estreito com o setor de Neurorradiologia do Centro de Ciências da Imagem do Hospital das Clínicas de Ribeirão Preto, através do desenvolvimento de projetos de pesquisa, reuniões clínicas e científicas regulares e atividades didáticas comuns. O setor inclui radiologia vascular, intervenção terapêutica, utiliza técnicas avançadas de ressonância magnética quantitativa, incluindo espectroscopia de prótons e laboratório para processamento de imagem.

\subsection{Pos-Graduação em Saúde Mental}

Historicamente, a criação do Programa de PósGraduação em Saúde Mental, em 1991, foi o marco da articulação entre as disciplinas Psiquiatria e Psicologia em torno de um projeto acadêmico e científico, liderado pelo Prof. Cláudio Roberto de Carvalho Rodrigues. Seu primeiro coordenador foi o Prof. Antonio Waldo Zuardi.

Concebido em modelo interdisciplinar, o programa se destina, desde sua origem, a alunos com graduação nas diversas áreas que atuam na saúde mental, como Medicina, Enfermagem, Psicologia, Serviço Social e Terapia Ocupacional. Seu principal objetivo é a formação de pesquisadores capacitados a conduzir projetos e a treinar novos pesquisadores em linhas de investigação, voltadas para a identificação e o contro- le de fatores de risco e condições de vulnerabilidade biológica e psicossocial para distúrbios psiquiátricos. Tendo como referência o adoecimento, a proposta contempla a prevenção, através de uma perspectiva evolutiva dos transtornos mentais e do estudo dos processos fundamentais das manifestações psicopatológicas.

O programa está ancorado em duas linhas de investigação: I. Fatores de risco, prevenção e intervenção em saúde mental, direcionada para a investigação de condições psicossociais, associadas à saúde e à doença mental; II. Fisiopatogenia dos transtornos psiquiátricos, orientada para a investigação de processos básicos de distúrbios mentais, bem como manifestações sintomáticas, condições envolvidas na formação dos sintomas e funcionamento de pessoas diagnosticadas. Na linha Fatores de risco, prevenção e intervenção em saúde mental inclui-se o estudo de temas, como: fatores psicossociais de risco e proteção a que estão expostos os indivíduos em desenvolvimento; indicadores de qualidade de vida, associados a condições físicas e à presença de enfermidade; modalidades de intervenção e abordagens terapêuticas. A linha de pesquisa Fisiopatogenia dos transtornos psiquiátricos focaliza distúrbios de ansiedade, esquizofrenia e distúrbios afetivos, combinando técnicas comportamentais avançadas e técnicas não invasivas, de estudo da morfologia e função do sistema nervoso central em seres humanos, que vêm adquirindo importância crescente no esclarecimento da fisiopatologia dos transtornos psiquiátricos.

Os grupos de pesquisa, vinculados ao programa mantêm intercâmbio ativo com pesquisadores da Universidade de São Paulo e de outras universidades no País e no Exterior. Esses grupos contam com o apoio de órgãos de fomento à pesquisa, como a FAPESP e o CNPq, através de bolsas de pesquisa, bolsas de pós-graduação e auxílios a projetos temáticos.

A interdisciplinaridade do programa se configura nos projetos temáticos, visando à obtenção de resultados conceitualmente coerentes sobre um tema de pesquisa. Tais projetos, que se desdobram em módulos logicamente interligados, permitem ao pósgraduando exercitar mais amplamente o raciocínio que fundamenta a busca do conhecimento científico, participando da construção dos nexos lógicos entre sua própria investigação e a dos outros pesquisadores da equipe. Essa formação é complementada com disciplinas metodológicas, voltadas para a especificidade da investigação na área. 
Essa breve reconstituição histórica mostra que o Departamento de Neurologia, Psiquiatria e Psicologia Médica consolidou suas atividades calcado em três pilares de atuação: a assistência, o ensino e a pesquisa. Os serviços assistenciais oferecidos pelo departamento caracterizam-se por atendimento multiprofissional, com a utilização das técnicas mais avançadas e disponíveis, servindo como referência especializada para diversos serviços da região. Essa diversidade de serviços assistenciais possibilitou que o departamento oferecesse um treinamento diferenciado tanto para alunos de graduação quanto para alunos vinculados a programas de residência médica e aprimoramento (pós-graduação lato sensu) de diferentes áreas. Nos últimos anos, houve um incremento da produção cien- tífica do departamento, caracterizada pela formação de um expressivo número de novos pesquisadores e pela publicação de trabalhos em periódicos especializados, tanto nacionais, como internacionais. A evolução recente dos programas de pós-graduação, oferecidos pelo departamento faculta uma visão otimista de seu desenvolvimento na primeira década do milênio.

\section{REFERÊNCIAS BIBLIOGRÁFICAS}

1 - LISON MP Ribeirão Preto. In: REIMÃO R \& ALONSO NIETO JL, orgs. História da Neurologia no Estado de São Paulo, Ed. Lemos, São Paulo, p. 139 -147, 1996.

2 - DEPARTAMENTO DE NEUROPSIQUIATRIAE PSICOLOGIAMÉDICA. Medicina, Ribeirão Preto, 25: 113-120, 1992. 